\title{
Aplicación móvil para mejorar la capacidad cognitiva en adultos mayores utilizando juegos mentales
}

Recibido: 15 Ago 2016 - Revisado: 30 Sep 2016

Aceptado: 30 Oct 2016 - Publicado: 30 Dic 2016

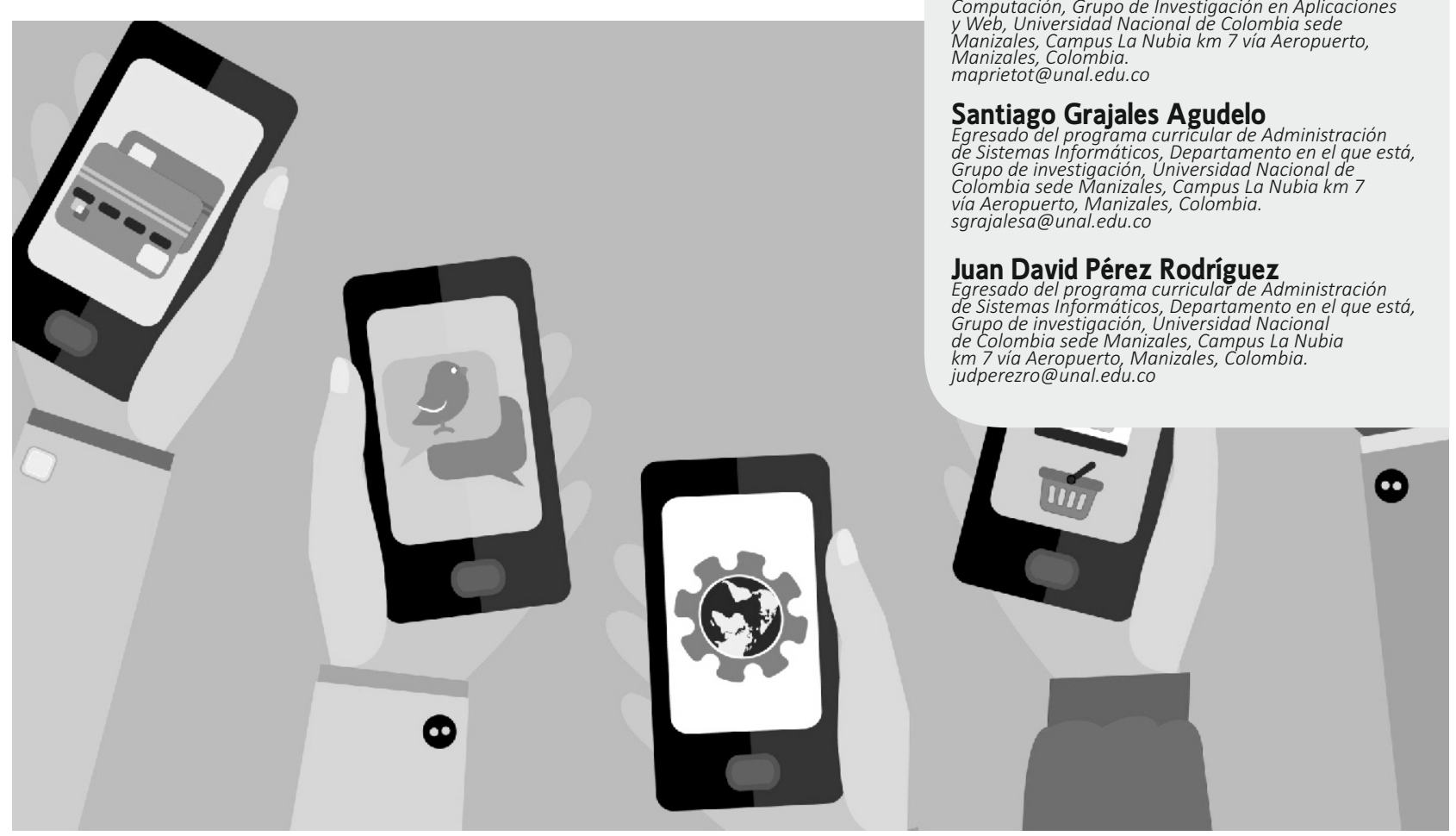

Resumen: Las tecnologías móviles pueden traer grandes beneficios para el desarrollo de una mejor calidad de vida de las personas, especialmente a una población tan vulnerable como los adultos mayores, los cuales debido a que presentan enfermedades y carencias propias de su edad. Además, debido a la revolución tecnológica que se experimenta actualmente, van quedando rezagados y presentan dificultades para aprender de estas innovaciones. Por tal razón, el objetivo de este estudio, es dar a conocer una iniciativa que pretende mejorar la capacidad cognitiva de ese grupo focal, a través del uso de tecnologías móviles. La aplicación desarrollada está compuesta de cuatro módulos: juegos mentales, calendario, noticias y tests mentales. Los resultados obtenidos en un grupo de adultos mayores corresponden a que muestran un gran interés en categorías como memoria, juegos y entrenamiento cerebral.

Palabras clave: aplicaciones móviles; adultos mayores; inclusión digital; juegos mentales; capacidad cognitiva.

Abstract: Mobile technologies can bring great benefits for the development of a better life quality for people, especially for a population as vulnerable as the elderly, who have diseases and deficiencies of their own age. In addition, due to the technological revolution that is currently experienced, they are lagging behind and present difficulties to learn from these innovations. For this reason, the objective of this study is to present an initiative that aims to improve the cognitive capacity of this focus group, using mobile technologies. The application developed is composed of four modules: mental games, calendar, news and mental tests. The results obtained in a group of older adults to show great interest in categories such as memory, games and brain training.

Key words: mobile applications; elderly; digital inclusion; mind games; cognitive capacity 


\section{INTRODUCCIÓN}

El número de personas mayores se está incrementando actualmente. El aumento de las enfermedades relacionadas con la edad como consecuencia del envejecimiento de la población mundial, ha hecho más evidente la necesidad de enfoques que permitan hacer frente a la disminución cognitiva (Morán, Meza-Kubo 2012).

Un adulto con problemas cognitivos tiene intacta la capacidad de aprender con facilidad, con la paciencia y el tiempo necesario para adaptar y asimilar el propio conocimiento.

En este escenario, las tecnologías móviles (Beato et al. 2007; Fraile et al. 2007) son de vitales y muy importantes para alcanzar los principales objetivos de la computación ubicua (Bardram et al. 2007; Hansen et al. 2006).

Las personas mayores pueden beneficiarse ampliamente al utilizar dispositivos móviles ya que estos les crean un sentido de autonomía hacia una vida independiente. A su vez, les pueden ayudar a desarrollar nuevos conocimientos, destrezas y seguridad en símismos (Azuddin et al. 2014).

Por lo tanto, es necesario desarrollar estrategias para modificar favorablemente las condiciones en las que los ancianos están viviendo, con el fin de prevenir la aparición o progresión del deterioro cognitivo.

Lo anterior se puede lograr a través de un concepto científico denominado "gimnasia cerebral" (Dennison and Dennison 1994) y un concepto tecnológico denominado «aplicación o aplicativo móvil» (Techopedia).

La fusión de estos elementos dará como resultado un modelo tecnológico que ayuda a entrenar las mentes de las personas adultas mayores a través de la tecnología inteligente como smartphones o tablets.
En la edad avanzada, conjuntamente con otros cambios fisiológicos, existe la tendencia de experimentar mermas en las funciones mentales. Algunos de estos cambios son debido a varias enfermedades o a procesos de envejecimiento irreversibles.

Sin embargo, muchos de estos cambios pueden impedirse o retrasarse llevando una vida activa y teniendo en cuenta varios factores como una dieta saludable, contactos sociales, aprendizaje continuo, buen estado físico, etc. (Consorcio de socios del Proyecto Mindwellness 2010).

Para hacer frente a esta problemática, se ha desarrollado BrainGym, una aplicación móvil con el fin de mejorar la capacidad cognitiva de las personas mayores. Ésta aplicación se evaluó utilizando técnicas subjetivas, mediante el uso de entrevistas y observación, para recoger la percepción de los participantes con respecto a la utilidad de la aplicación, facilidad de uso y la experiencia del usuario.

El artículo está organizado de la siguiente manera: la sección 2 presenta trabajos relacionados con el área de investigación, la sección 3 presenta la metodología utilizada para el desarrollo de la aplicación propuesta; la sección 4 describe la aplicación desarrollada y sus módulos; la sección 5 presenta la validación hecha con un par de personas mayores. Finalmente, la sección 6 presenta las conclusiones y direcciones de trabajo futuro.

\section{TRABAJOS RELACIONADOS}

En la literatura e internet se encuentran numerosas aplicaciones orientadas a las personas mayores.

En la Tabla 1, se describen algunas de las más llamativas. Las aplicaciones no solo están centradas para este tipo de población, también incluyen juegos y 
actividades para ayudar en procesos de estimulación como la memoria.

En la Tabla 1, se observa que estas aplicaciones móviles están principalmente orientadas al entrenamiento cognitivo (memoria, concentración, lógica), lenguaje, usabilidad (visión, reconocimiento de voz, interfaz), ejercicios y terapias físicas, y consulta de datos médicos y clínicos.

Las principales debilidades desde el punto de vista del público objetivo es la utilización del idioma inglés, algunas no son de libre distribución, se enfocan en áreas específicas y a resolver problemas muy concretos.

Respecto a juegos de entrenamiento mental para personas mayores online, en la Tabla 2 se presenta algunos ejemplos.

\section{METODOLOGÍA}

La metodología utilizada para el desarrollo de la aplicación web consta de los siguientes pasos y está basada en el enfoque Mobile-D (Abrahamsson 2004).

- Fase de exploración: donde se definió el alcance del proyecto, se aplicó una encuesta con el fin de identificar las necesidades de una comunidad de adultos mayores y se realizó un análisis inicial de requisitos.

- Fase de desarrollo de la aplicación móvil: a través de varias iteraciones se inicializa, se produce y estabiliza la aplicación móvil.

- Fase de pruebas y validación de la aplicación desarrollada: se realizaron pruebas de usuario final con varios sujetos de estudio.

A continuación se presentarán detalles de cada una de las fases.
Tabla 1. Aplicaciones móviles orientadas a personas mayores

\begin{tabular}{|c|c|c|}
\hline Aplicación & Objetivo & Funcionalidad \\
\hline Medisafe & $\begin{array}{l}\text { Ayudar en la } \\
\text { toma de } \\
\text { medicamentos }\end{array}$ & Alertas por SMS o email. \\
\hline $\begin{array}{l}\text { Fit Brains } \\
\text { Trainer }\end{array}$ & $\begin{array}{l}\text { Estimular y } \\
\text { desafiar al } \\
\text { cerebro }\end{array}$ & $\begin{array}{l}\text { Juegos: } \\
\text { Video-espaciales, } \\
\text { concentración, memoria, } \\
\text { lógica y lenguaje }\end{array}$ \\
\hline $\begin{array}{l}\text { A Story } \\
\text { Before Bed }\end{array}$ & $\begin{array}{l}\text { Contar cuentos } \\
\text { antes de ir a } \\
\text { dormir }\end{array}$ & $\begin{array}{l}\text { Funciones: } \\
\text { - Selección de cuentos } \\
\text { gráficos } \\
\text { - Grabación de lectura por } \\
\text { webcam }\end{array}$ \\
\hline Mimov & $\begin{array}{l}\text { Servicio de tele - } \\
\text { asistencia familiar }\end{array}$ & $\begin{array}{l}\text { Funciones: } \\
\text { - Localización de usuarios } \\
\text { - Acceso a últimas } \\
\text { posiciones } \\
\text { - Botón de llamada }\end{array}$ \\
\hline $\begin{array}{l}\text { BIG } \\
\text { Launcher }\end{array}$ & $\begin{array}{l}\text { Ayudar a } \\
\text { personas mayores } \\
\text { y personas con } \\
\text { baja visión }\end{array}$ & $\begin{array}{l}\text { Funciones: } \\
\text { - Interfaz simple con } \\
\text { pocos y grandes iconos } \\
\text { Alarmas, contactos, } \\
\text { Ilamadas, SMS }\end{array}$ \\
\hline Cardiograph & $\begin{array}{l}\text { Medir la } \\
\text { frecuencia } \\
\text { cardiaca }\end{array}$ & $\begin{array}{l}\text { Funciones: } \\
\text { - Cámara para fotos de } \\
\text { yema del dedo y calcular } \\
\text { ritmo cardiaco } \\
\text { Registro de datos } \\
\text { históricos }\end{array}$ \\
\hline $\begin{array}{l}\text { Automesure } \\
\text { tensionnelle }\end{array}$ & $\begin{array}{l}\text { Realizar } \\
\text { seguimiento de la } \\
\text { presión arterial y } \\
\text { monitorizar } \\
\text { resultados a largo } \\
\text { plazo }\end{array}$ & $\begin{array}{l}\text { Funciones: } \\
\text { - Programación de } \\
\text { mediciones } \\
\text { - Cálculo automático } \\
\text { - Informes históricos } \\
\text { - Representación gráfica } \\
\text { de resultados (PDF, } \\
\text { email) }\end{array}$ \\
\hline $\begin{array}{l}\text { Motion } \\
\text { Doctor }\end{array}$ & $\begin{array}{l}\text { Realizar terapias } \\
\text { físicas }\end{array}$ & $\begin{array}{l}60 \text { videos categorizados: } \\
\text { - Partes del cuerpo } \\
\text { - Actividades } \\
\text { - Deportes } \\
\text { Ocupaciones }\end{array}$ \\
\hline $\begin{array}{l}\text { Dragon } \\
\text { Dictation }\end{array}$ & $\begin{array}{l}\text { Reconocimiento } \\
\text { de voz }\end{array}$ & $\begin{array}{l}\text { Hablar para: } \\
\text { - Dictar notas } \\
\text { - Enviar actualizaciones a } \\
\text { Facebook o Twitter } \\
\text { - Enviar emails o SMS }\end{array}$ \\
\hline Memory & $\begin{array}{l}\text { Ejercitar la } \\
\text { memoria con } \\
\text { tarjetas y niveles } \\
\text { de dificultad }\end{array}$ & $\begin{array}{l}\text { Juegos } \\
\text { - Sopa de letras } \\
\text { - Quiz de logos } \\
\text { - Ahorcado } \\
\text { - Sopa de letras }\end{array}$ \\
\hline
\end{tabular}




\begin{tabular}{|lll|}
\hline Lumosity & Entrenar & Funciones: \\
& memoria, & - Diagnóstico inicial \\
& atención, & - Sesiones diarias \\
& velocidad, y & - Seguimiento de \\
& resolución de & puntuaciones \\
& problemas & \\
\hline
\end{tabular}

Tabla 2. Aplicaciones web orientadas al entrenamiento cognitivo de personas mayores

\begin{tabular}{|lll|}
\hline Aplicación & \multicolumn{1}{c|}{ Objetivo } & \multicolumn{1}{c|}{ Funcionalidad } \\
\hline Simon & Ejercitar la memoria a & Juego con colores y \\
Dice & través de pasos en una & audio para memorizar \\
Online & secuencia & \\
Plus & Proporcionar juegos & - Juegos de memoria \\
memoria & online diseñados para & online. \\
& ejercitar la mente & $\begin{array}{c}\text { - Juegos en texto } \\
\text { escrito en formato } \\
\end{array}$ \\
& & PDF \\
\hline
\end{tabular}

\subsection{Fase de exploración}

Para la realización de esta etapa, se realizó una encuesta en un hogar para personas mayores con el fin de identificar sus principales problemas, las preferencias, los gustos, y requerimientos de la comunidad adulta. La encuesta se aplicó a una muestra pequeña de la población de edad avanzada, 24 personas en total, que se encontraban en los centros de salud privados en la ciudad de Manizales (Caldas).

La encuesta constaba de 17 preguntas y se centraron principalmente en saber cómo ellas manejan la tecnología móvil y cómo aplicarla para mejorar su calidad de vida.

La Tabla 3 muestra las preguntas de la encuesta y los resultados correspondientes, de donde se pueden concluir los resultados que se mencionan a continuación.

- Todas las personas tienen algún grado de educación de la primaria a la universidad. Esto revela que las habilidades cognitivas pueden ser aprovechadas para mejorar su salud mental mediante la formación y juegos.
- Hay una baja proporción de personas de edad avanzada que utilizan tecnología móvil, pero las preferencias por los teléfonos 3G, ayudan a mitigar un poco el problema, que radica en la adquisición y la enseñanza en utilización de los dispositivos.

- Las personas han instalado en sus dispositivos aplicaciones como las de entrenamiento cognitivo, juegos mentales y libros.

- Las personas mayores están seguras de que pueden entrenar sus cerebros mediante un dispositivo móvil.

Tabla 3. Encuesta realizada a población sujeto del estudio

\begin{tabular}{|c|c|}
\hline Pregunta & Resultados \\
\hline Sexo & $\begin{array}{ll}\text { - } & \text { Hombres: } 9 \\
\text { - } & \text { Mujeres: } 15\end{array}$ \\
\hline Edad & $\begin{array}{ll}\text { - } & \text { 50-60 años: } 9 \\
\text { - } & 61-70 \text { años: } 10 \\
\text { - } & 70+: 5\end{array}$ \\
\hline Nivel educativo & $\begin{array}{ll}\text { - } & \text { Primaria: } 4 \\
\text { - } & \text { Secundaria: } 7 \\
\text { - } & \text { Técnico/Tecnológico: } 7 \\
\text { - } & \text { Universidad: } 6\end{array}$ \\
\hline $\begin{array}{l}\text { ¿Tiene teléfono } \\
\text { móvil? }\end{array}$ & $\begin{array}{ll}\text { - } & \text { Si: } 23 \\
\text { - } & \text { No: } 1\end{array}$ \\
\hline $\begin{array}{l}\text { ¿Tiene acceso a } \\
\text { internet desde el } \\
\text { dispositivo? }\end{array}$ & $\begin{array}{ll}\text { - } & \text { Si: } 8 \\
\text { - } & \text { No: } 5 \\
\text { - } & \text { NS/NR: } 11\end{array}$ \\
\hline $\begin{array}{l}\text { Tecnología } \\
\text { utilizada: }\end{array}$ & $\begin{array}{ll}\text { - } & 3 G: 8 \\
\text { - } & 4 G: 16\end{array}$ \\
\hline $\begin{array}{l}\text { ¿Ha visitado una } \\
\text { app store? }\end{array}$ & $\begin{array}{ll}\text { - } & \text { Si: } 1 \\
\text { - } & \text { No: } 8 \\
\text { - } & \text { NS/NR: } 15\end{array}$ \\
\hline ¿Ha instalado apps? & $\begin{array}{ll}\text { - } & \text { Si: } 1 \\
\text { - } & \text { No: } 8 \\
\text { - } & \text { NS/NR: } 14\end{array}$ \\
\hline $\begin{array}{l}\text { ¿Sabe utilizar otros } \\
\text { dispositivos móviles } \\
\text { diferentes al } \\
\text { teléfono móvil? }\end{array}$ & $\begin{array}{ll}\text { - } & \text { Tablet: } 7 \\
\text { - } & \text { Computador: } 9 \\
\text { - } & \text { Otro: } 4 \\
\text { - } & \text { Ninguno: } 10\end{array}$ \\
\hline
\end{tabular}




\begin{tabular}{|c|c|}
\hline $\begin{array}{l}\text { Tipo de } \\
\text { aplicaciones } \\
\text { utilizadas }\end{array}$ & $\begin{array}{ll}\text { - } & \text { Salud: } 4 \\
\text { - } & \text { Entrenamiento cognitivo: } 7 \\
\text { - } & \text { Juegos: } 7 \\
\text { - } & \text { Juegos mentales: } 6 \\
\text { - } & \text { Libros: } 7 \\
\text { - } & \text { Ninguno: } 1\end{array}$ \\
\hline $\begin{array}{l}\text { ¿Cree que a través } \\
\text { de un dispositivo } \\
\text { móvil puede } \\
\text { entrenar su } \\
\text { cerebro? }\end{array}$ & $\begin{array}{ll}\text { - } & \text { Si: } 22 \\
\text { - } & \text { No: } 2\end{array}$ \\
\hline $\begin{array}{l}\text { Cosas que más } \\
\text { frecuentemente } \\
\text { olvida }\end{array}$ & $\begin{array}{ll}\text { - } & \text { Nombre y apellidos: } 5 \\
\text { - } & \text { Teléfonos: } 6 \\
\text { - } & \text { Direcciones: } 12 \\
\text { - } & \text { Actividades diarias: } 3 \\
\text { - } & \text { Nada: } 2\end{array}$ \\
\hline
\end{tabular}

\subsection{Fase de desarrollo de la aplicación móvil}

La herramienta denominada Mobincube (2015) fue utilizada para desarrollar la aplicación móvil. Es un editor muy intuitivo que permite la creación de apps nativas para smartphones y tablets. Además, ofrece una solución web.

Los requerimientos de usabilidad, eficiencia y portabilidad de la aplicación móvil fueron especificados como se menciona a continuación.

- De usabilidad: la aplicación puede ser utilizada por usuarios sin mucha experiencia en dispositivos móviles, debido a que son personas sin acceso a dichos dispositivos. Por lo tanto, la aplicación debe ser amigable, simple y de fácil uso.

- De eficiencia: la aplicación debe tener acceso rápido a todas sus funcionalidades y proporcionar la mejor experiencia para el manejo de la aplicación.

- De portabilidad: la aplicación debe ser diseñada para dar al usuario opciones de inter-operabilidad al ser instalada y ejecutada en smartphone $y$ tablets, con arquitectura Android y arquitectura iPhone/iPadiOS.

\subsection{Fase de pruebas y validación}

El proceso de validación de la aplicación inició con el consentimiento de dos personas mayores quienes estuvieron de acuerdo en realizar pruebas de la aplicación. Las dos personas eran mujeres, quienes leyeron el manual del usuario de la aplicación y utilizaron una tablet para consultar y ejecutar los diferentes módulos de la aplicación.

El proceso de validación finalizó con la realización de entrevistas y observación del uso de la aplicación como técnicas de evaluación.

\section{DESCRIPCIÓN DE LA SOLUCIÓN PROPUESTA}

El objetivo de la aplicación desarrollada es minimizar los daños físicos y psicológicos que el paso del tiempo ha traído en los cerebros de la población mayor. Esto se logra al mismo tiempo de que estas personas se entretienen: a través de juegos y actividades, ayudan a entrenar sus capacidades cognitivas, que se han degradado por las afecciones naturales que se presentan en el transcurso de la vida.

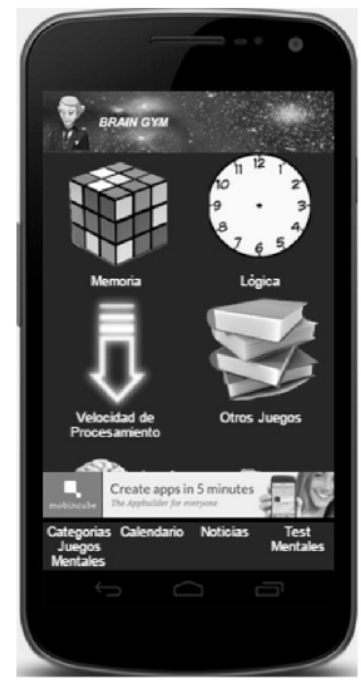

Figura 2. Módulo de juegos mentales. Este módulo incluye juegos clasificados en las categorías de: memoria, lógica, velocidad de procesamiento, entrenamiento corporal y otros juegos. 


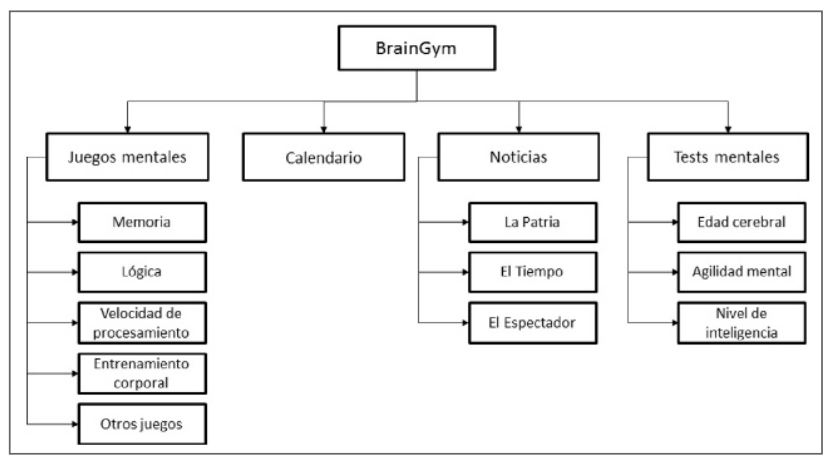

Figura 1. Estructura general de BrainGym. La estructura de la aplicación móvil está conformada por cuatro módulos: juegos mentales, calendario, noticias y tests mentales.

Al iniciar la aplicación, la primera pantalla que aparece al acceder, presenta un ícono representativo de la gimnasia cerebral con el propósito de despertar la curiosidad en el usuario.

Allí mismo, se implementa un fondo blanco ya que según la teoría del color, brinda sensación de calma y tranquilidad, originando la impresión de confianza en el adulto mayor; todo con el objetivo de minimizar el rechazo o temor al manejo e interacción, tanto con la aplicación como con el dispositivo móvil que utiliza.

(a) Módulo de juegos mentales: presenta un menú tipo tabla, con una lista de categorías relacionadas con juegos de entrenamiento y gimnasia cerebral. Tiene las siguientes categorías que se ampliarán a continuación: memoria, lógica, velocidad de procesamiento, entrenamiento corporal y otros juegos (ver Figura 2).

- Memoria: en esta categoría se incluyen juegos cuyo objetivo es potenciar la memoria a corto y largo plazo, también que ayudan a estimular la inteligencia.

Los adultos mayores tienden a perder su memoria fotográfica o visual, y con la ayuda de estos juegos podrán retardar esa pérdida.
Además, a través de ellos, los usuarios contarán con la posibilidad de recordar más fácilmente experiencias pasadas y que han retenido en sus memorias a lo largo del tiempo. También conocimientos que hayan adquirido previamente, pero por falta de práctica y entrenamiento cerebral, han quedado alojados en lo profundo de sus memorias.

- Lógica: estos juegos brindan la posibilidad de aumentar las habilidades cognitivas y activar el procesamiento lógico, debido a que sus reglas requieren de un razonamiento lógico previo para identificar cuál es el objetivo a cumplir y/o realizar en cada competencia.

- Velocidad de procesamiento: en esta categoría se incluyen juegos de entrenamiento cerebral, los cuales tienen como propósito conservar la capacidad de detectar, procesar y emitir determinada cantidad de información por unidad de tiempo.

- Entrenamiento corporal: en esta categoría se pueden visualizar ejercicios corporales que son bastante simples y que al realizarlos de manera constante y cotidiana, favorecen al entrenamiento de ciertas actividades cerebrales como concentración, coordinación, comprensión lectora, motricidad fina, entre otras, (que disminuyen con el paso de los años en los adultos mayores). Estas actividades ayudan además a ejercitar algunos músculos y articulaciones, apoyando así el movimiento corporal de estas personas.

- Otros juegos: en este segmento se incluyen tanto de entrenamiento cerebral como de ocio para el ejercicio de los adultos mayores: sopas de letras, crucigramas y otros; su objetivo es ayudar a fijar la concentración en juegos que aunque son tradicionales, mantienen el cerebro activo y permiten mitigar de cierta forma, los efectos causados por la vejez. 


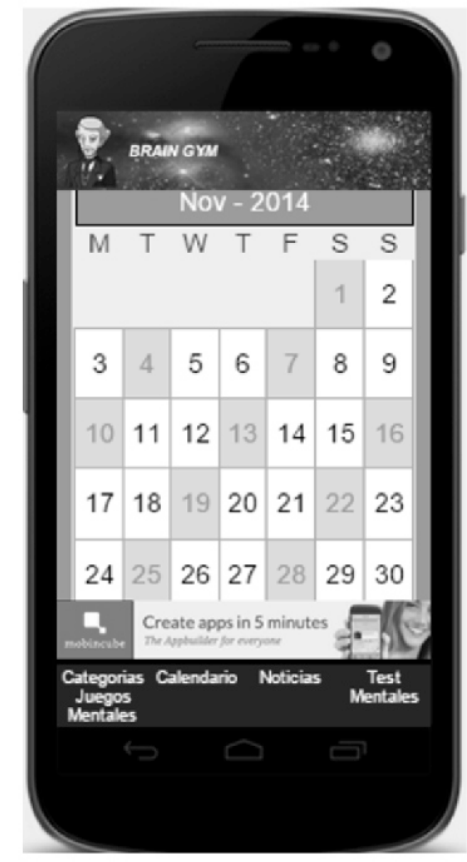

Figura 3. Módulo de calendario. Este módulo permite programar la realización de actividades por fechas seleccionadas a partir de un calendario presentado.

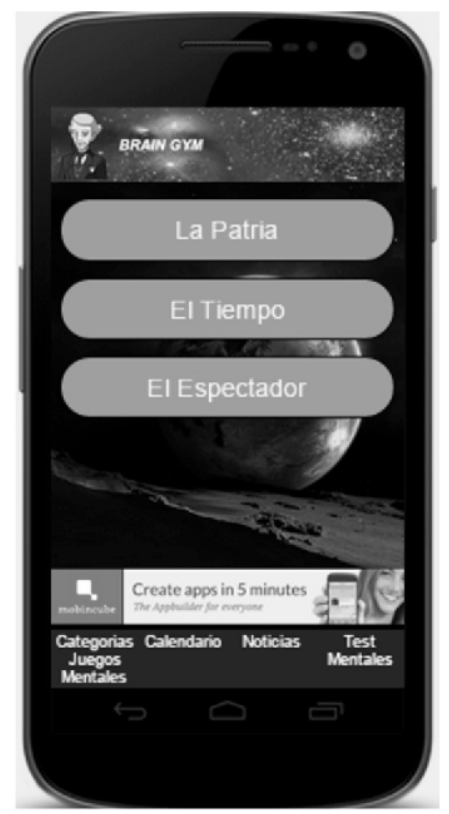

Figura 4. Módulo de noticias. Este módulo permite consultar algunos periódicos del nivel nacional para su consulta y lectura. (b) Módulo de calendario: permite el entrenamiento calendarizado para que el usuario, además de entrenar en forma aleatoria, pueda hacerlo cada tres días de forma diversa. Esto posibilita que afiance múltiples acciones cerebrales, evitando así que se ejerciten unas actividades más que otras. El usuario debe ubicarse en la fecha de calendario actual y si está resaltada con color azul al tocar su casilla, inmediatamente se ejecuta el juego destinado para ese día específico (ver Figura 3).

Figura 4. Módulo de noticias. Este módulo permite consultar algunos periódicos del nivel nacional para su consulta y lectura.(c) Módulo de noticias: presenta enlaces a algunos periódicos y revistas nacionales, con el propósito de incentivar la lectura digital. A través del hábito de la lectura, se entrena el cerebro para que adquiera conocimientos, lo que contribuye a la capacidad cognitiva y a la memoria a largo y corto plazo. (ver Figura 4).

(d) Módulo de tests mentales: incluye una serie de tests o pruebas cerebrales para medir el rendimiento y progreso del usuario a través del desarrollo de los juegos de gimnasia cerebral (ver Figura 5).

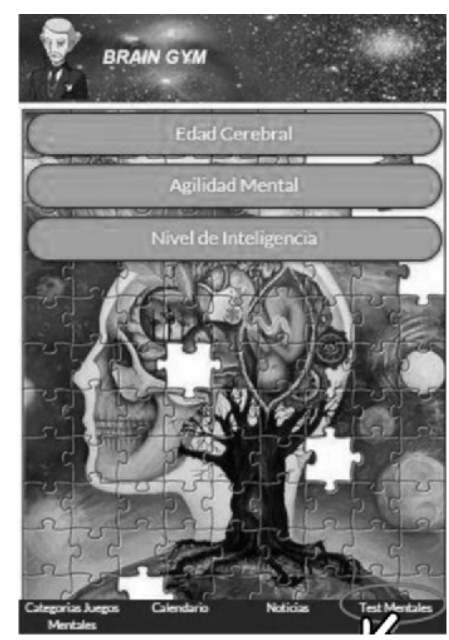

Figura 5. Módulo de tests mentales. Este módulo permite la ejecución de pruebas para medir su desempeño en diversos juegos como: Edad cerebral, agilidad mental y nivel de inteligencia. 


\section{VALIDACIÓN}

La validación fue realizada mediante la ejecución de las etapas que se mencionan a continuación.

Debido a razones de disponibilidad de los sujetos y para cumplir las restricciones de tiempo del proyecto, se seleccionaron dos mujeres del conjunto poblacional al que se aplicó la encuesta.

Estas personas leyeron cuidadosamente el manual de BrainGym; luego se discutió con ellas el contenido del manual para verificar su comprensión. Después, se les entregó una tablet con sistema operativo Android que tenía instalada la aplicación.

Las tablets fueron utilizadas durante la validación debido a que son dispositivos con un buen tamaño y resolución de pantalla para una mejor lectura y comprensión de íconos, teniendo en cuenta los problemas visuales presentados por las personas sujetos de las pruebas (Jayroe and, Wolfram 2011).

Una de las mujeres utilizó rápidamente la aplicación debido a que tenía conocimiento previo acerca del uso de apps. Por otro lado, la segunda mujer no tenía experiencia al respecto, así que fue necesario explicarle repetidamente cómo acceder a los módulos, de manera que después de varios intentos, pudo memorizar los pasos.

Durante el uso de la aplicación por ambas mujeres, ninguna recordaba cómo acceder a varios de los módulos. Por lo tanto, se procedió a explicarles el manual paso a paso, mientas interactuaban con la aplicación.

Se les orientó para acceder a cada uno de los módulos componentes de la aplicación, en el orden que se presentó en la Figura 1.
Fig. 5. Módulo de tests mentales. Este módulo permite la ejecución de pruebas para medir su desempeño en diversos juegos como: Edad cerebral, agilidad mental y nivel de inteligencia.

A medida que se observaba cómo se desempeñaban en los diferentes módulos de la aplicación, se llevaba un registro de los principales éxitos e inconvenientes presentados con su uso.

Finalmente, a partir de entrevistas, se evidenciaron los siguientes comentarios por parte de los sujetos de prueba.

- Respecto a los juegos y formas de entrenamiento, manifestaron un gran interés en módulos como memoria, otros juegos y ejercicio físico.

- Argumentaron que la aplicación proporciona acceso a periódicos de una forma fácil.

- En relación a las pruebas de desempeño mental, sintieron que la aplicación les permitía conocer su progreso mientras desarrollaban las diferentes actividades incluidas.

Además, el comportamiento de los sujetos concuerda con los resultados presentados por diversos autores (Kobayashi et al. 2011), donde las interfaces móviles con touchscreen no son difíciles de utilizar por personas mayores, y se muestra un significativo desempeño en pinchar y soltar.

\section{CONCLUSIONES}

Las sociedades actuales deben entender que la población de edad avanzada está rezagada tecnológicamente. Por lo tanto, es una obligación brindarles ayuda en el proceso de inclusión tecnológica, el cual puede ser realizado a través del uso activo de aplicaciones móviles.

A medida que la población de mayor edad se vuelve demasiado resistente al cambio, algunas estrategias se deben implementar en el hogar y a nivel 
profesional, con el fin de hacer un acompañamiento en la utilización de estos dispositivos de manera eficaz.

El uso de estas aplicaciones mostrará las ventajas que pueden aportar buscando aumentar la calidad de vida de las personas mayores, además de ser medios de entretenimiento para evitar la monotonía.

No sólo los amigos, la familia o personas cercanas a los miembros de esta población, deben tomar la iniciativa para apoyarlos en este proceso cognitivo; sino también los gobiernos de las distintas entidades territoriales deben obtener y distribuir los recursos necesarios para poner en práctica estrategias o planes, que ayuden a fomentar la formación a través gimnasia cerebral.

Tabla 5. Comparativo de aplicaciones y sitios web relacionados

\begin{tabular}{|c|c|c|c|c|c|}
\hline Aplicación & Memoria & Lógica & Velocidad & $\begin{array}{l}\text { Actividad } \\
\text { física }\end{array}$ & Lenguaje \\
\hline Medisafe & $x$ & - & - & - & - \\
\hline $\begin{array}{l}\text { Fit Brains } \\
\text { Trainer }\end{array}$ & $x$ & $x$ & - & - & $x$ \\
\hline $\begin{array}{l}\text { A Story } \\
\text { Before } \\
\text { Bed }\end{array}$ & - & - & - & - & $x$ \\
\hline Mimov & $x$ & - & - & - & - \\
\hline $\begin{array}{l}\text { BIG } \\
\text { Launcher }\end{array}$ & - & - & - & - & $x$ \\
\hline $\begin{array}{l}\text { Cardiogra } \\
\text { ph }\end{array}$ & - & - & - & $x$ & - \\
\hline $\begin{array}{l}\text { Automes } \\
\text { ure } \\
\text { tensionne } \\
\text { lle }\end{array}$ & - & - & - & $x$ & - \\
\hline $\begin{array}{l}\text { Motion } \\
\text { Doctor }\end{array}$ & - & - & - & $x$ & - \\
\hline $\begin{array}{l}\text { Dragon } \\
\text { Dictation }\end{array}$ & - & - & - & - & $x$ \\
\hline Memory & - & - & - & - & $x$ \\
\hline Lumosity & $x$ & - & $x$ & $x$ & - \\
\hline $\begin{array}{l}\text { Simon } \\
\text { Dice } \\
\text { Online }\end{array}$ & $x$ & - & - & - & - \\
\hline $\begin{array}{l}\text { Plus } \\
\text { memoria }\end{array}$ & $x$ & - & - & - & - \\
\hline BrainGym & $x$ & $x$ & $x$ & $x$ & $x$ \\
\hline
\end{tabular}

Así, con el acceso a estos dispositivos de ancianos pobres o aquellos que lo tienen limitado, se podrá mejorar su calidad de vida y cumplir los requisitos de inclusión digital. La población mayor debe apoyarse continuamente con instructores capacitados para tal fin.

Estas personas a través de dispositivos móviles estarán asimilando y almacenando información en diferentes grados. Con el tiempo, esta actividad se vuelve frecuente, lo que permite una interacción más amplia con los dispositivos.

La aplicación BrainGym ofrece un conjunto de juegos orientados a apoyar las capacidades cognitivas de las personas mayores.

En la Tabla 5, se presenta una comparación de la aplicación desarrollada con las referidas en los trabajos relacionados, los cuales están dispersos en las categorías de memoria, lógica, velocidad, actividad física y lenguaje. Las categorías más cubiertas son las orientadas a la memoria y el lenguaje. Muy pocas propuestas apuntan a la lógica y la velocidad.

La importancia de BrainGym radica en abarcar juegos y tests orientados a todas esas categorías para fortalecer el entrenamiento cognitivo.

Este trabajo tiene un impacto bastante alto a nivel regional y nacional, debido a que está enfocado en un grupo de usuarios muy particular, las personas de tercera edad.

La aplicación desarrollada ha determinado que se pueden ofrecer herramientas tecnológicas a ese nicho para ayudar a fortalecer y mantener habilidades cognitivas. Las principales fortalezas de la aplicación desarrollada son su grado de dificultad (no es muy alto para el público objetivo), su interfaz gráfica es simple y que cuenta con un conjunto altamente versátil de juegos y test mentales, que contribuyen al entrenamiento neurocognitivo. 
TRABAJO FUTURO Consistirá en incrementar la funcionalidad de la aplicación móvil para generar estadísticas de las pruebas realizadas.

Respecto a nuevas funcionalidades, se requiere que la aplicación permita que los usuarios establezcan objetivos para mejorar sus habilidades cognitivas y realizar el respectivo seguimiento su cumplimiento.

Además, es prioritario realizar experimentos con una muestra más grande y concretar asesorías de expertos para generar indicadores que permitan medir el mejoramiento en la capacidad cognitiva de manera progresiva.

Por último, se requiere un estudio más exhaustivo sobre el uso de este tipo de herramientas como los propuestos por varios autores (Abd-Malik et al. 2013; Zainal et a.l 2013).

Agradecimientos Este trabajo está enmarcado dentro del Proyecto de Fortalecimiento del Grupo de Aplicación y Herramientas Web de la Universidad Nacional de Colombia, sede Manizales (32087), dentro de las líneas de investigación en aplicaciones móviles y educación especial.

\section{REFERENCIAS}

Abd-Malik, S., Azuddin, M. (2013). Mobile technology for older people: Use of personas. International Conference on Research and Innovation in Information Systems (ICRIIS), pp. 97-101.

Abrahamsson, P., Hanhineva, A., Hulkko, H., lhme, T., Jäälinoja, J., Korkala, M., Koskela, J., Kyllönen, P., Salo, O. (2004). Mobile-D: an agile approach for mobile application development. Companion to the 19th annual ACM SIGPLAN conference on Object oriented programming systems, languages, and applications. pp. 174-175.

Automesure tensionnelle. Recuperado de http://www.ticsalut.cat/observatori/es_apps/11 7/automesure-tensionnelle

Azuddin, M., Abd-Malik, S., Abdullah, L.M., Mahmud, M. (2014). Older people and their use of mobile devices: Issues, purpose and context. 5th International Conference on Information and Communication Technology for The Muslim World (ICT4M). pp. 1-6.

A Story Before Bed. Recuperado de http://www.astorybeforebed.com/

Bardram, J.E., Baldus, H., Favela, J. (2007). Pervasive computing in hospitals. Pervasive Computing in Healthcare. (pp. 49- 77). Boca Ratón: CRC Press.

Beato, M.E., et al. (2008). Mobile devices: Practical cases on this new applications platform. In: Intl. workshop on practical applications of agents and multiagent Systems, IEEE Latin 
America Transactions, Vol 6, No. 6, (pp. 231-240). Salamanca.

BIG Launcher. Recuperado de http://biglauncher.com/home/es/

Cardiograph. Recuperado de http://macropinch.com/cardiograph/

Consorcio de socios del Proyecto Mindwellness. (2010). Manual sobre el entrenamiento mental de las personas mayores de 50 años. Recuperado d

http://masvida50.com/juegos/documen/Handb ook-ES.pdf

Dennison, P.E., Dennison, G.E. (1994). Brain Gym. Teachers Edition. Ventura: Edu-Kinesthetics.

Dragon Dictation. Recuperado de http://www.nuance.com/for-individuals/mobileapplications/dragon-dictation/index.htm

Fit Brains Trainer. Recuperado de http://www.fitbrains.com/

Fraile, J.A., Delgado, M., Sánchez, M.A., Beato, M.E. (2007). In: Intl. Workshop on Practical Applications of Agents and Multiagent Systems. The UPSA Mobile Information System MoviUPSA. (pp. 221-230). Salamanca.

Hansen, T.R., Bardram, J.E., Soegaard, M. (2006). Moving out of the lab: Deploying Pervasive Technologies in a hospital. IEEE Pervasive Computing, 5(3), 24-31.

Jannenga, H. Motion Doctor Develops iPad app designed for Physical Therapy home exercise instruction. Recuperado de https://www.webpt.com/blog/post/motiondoctor-develops-ipad-app-designed-physicaltherapy-home-exercise-instruction

Jayroe, T. J., Wolfram, D. (2011). Internet searching, tablet technology and older adults. Proceedings of the American Society for Information Science and Technology, 49(1).

Kobayashi, M., Hiyama, A., Miura, T., Asakawa, C., Hirose, M., Ifukube, T. (2011). Elderly User Evaluation of Mobile Touchscreen Interactions, Lecture Notes in Computer Science on HumanComputer Interaction. INTERACT 2011, 6946, 8399.

Luminosity. Recuperado de http://www.lumosity.com/

MediaSafe. Recuperado de

http://www.medisafe.com/

Memory. Recuperado de

https://play.google.com/store/apps/details?id=c om.berniiiiiiii.logomatchup

Mimov. Recuperado de

http://www.mimov.es/pages/servicios_mimov.p hp

Mobincube. Web site. (205) Recuperado de http://mobincube.com

Morán, A.L., Meza-Kubo, V. (2012). User experience of elders and relatives in a collaborative cognitive stimulation tool. In Ambient Assisted Living and Home Care, Lecture Notes in Computer Science. 4th International Workshop, IWAAL 2012, Vitoria-Gasteiz, Spain, 


\section{Ingenierías: $:$.}

December 3-5, 2012. ProceedingsVol 7657, Techopedia. What is a mobile app? Recuperado Springer, 287-294. de

http://www.techopedia.com/definition/2953/m PIus Memoria. Recuperado de https://sites.google.com/site/tecnicasdeestudio ebs2013/plus-memoria obileapplication-mobile-app

Zainal, A., Abdul-Razak, F.H., Akma-Ahmad, N. (2013). Older People and the Use of Mobile

Simon Dice Online. Recuperado de http://www.juegos-play.com/simon-dice Phones: An Interview Study. 2013 International Conference on Advanced Computer Science Applications and Technologies (ACSAT), pp. 390395. 